\title{
Dynamic Performance of Unimorph Piezoelectric Bending Actuators
}

DOI:

$10.1177 / 0959651814552810$

\section{Document Version}

Accepted author manuscript

Link to publication record in Manchester Research Explorer

\section{Citation for published version (APA):}

Nabawy, M. R. A., Parslew, B., \& Crowther, W. J. (2014). Dynamic Performance of Unimorph Piezoelectric Bending Actuators. Institution of Mechanical Engineers. Proceedings. Part l: Journal of Systems and Control Engineering, 229(2), 118-129. https://doi.org/10.1177/0959651814552810

\section{Published in:}

Institution of Mechanical Engineers. Proceedings. Part I: Journal of Systems and Control Engineering

\section{Citing this paper}

Please note that where the full-text provided on Manchester Research Explorer is the Author Accepted Manuscript or Proof version this may differ from the final Published version. If citing, it is advised that you check and use the publisher's definitive version.

\section{General rights}

Copyright and moral rights for the publications made accessible in the Research Explorer are retained by the authors and/or other copyright owners and it is a condition of accessing publications that users recognise and abide by the legal requirements associated with these rights.

\section{Takedown policy}

If you believe that this document breaches copyright please refer to the University of Manchester's Takedown Procedures [http://man.ac.uk/04Y6Bo] or contact uml.scholarlycommunications@manchester.ac.uk providing relevant details, so we can investigate your claim.

\section{OPEN ACCESS}




\title{
Dynamic Performance of Unimorph Piezoelectric Bending Actuators
}

\author{
Mostafa R. A. Nabawy, Ben Parslew and William J. Crowther \\ School of Mechanical, Aerospace and Civil Engineering, The University of Manchester, \\ Manchester, UK
}

\begin{abstract}
Piezoelectric bending actuators utilise the inverse piezoelectric effect to convert input electric energy to useful mechanical work, and are capable of producing relatively large bending deflections at low voltage. A comprehensive analytical model of the dynamic electromechanical behaviour of a unimorph piezoelectric actuator has been developed and successfully validated against experimental data. The model provides a mapping between force, displacement, voltage and charge. Damping is modelled using experimental data. Experimental validation is based on measurement of mode shape and frequency response of a series of unimorph beams of varying length but of the same thickness and material. The experimental frequency response is weakly nonlinear with excitation voltage, with a reduction in natural frequency and increase in damping with increasing excitation amplitude. An expression for the ElectroMechanical Coupling Factor (EMCF) has been extracted from the analytical model and is used as the objective for parametric design studies. The design parameters are thickness ratio and Young's modulus ratio of the elastic and piezoceramic layers, and the piezoelectric constant $k_{31}$. The operational design point is defined by the damping ratio. It is found that the relative variation of the EMCF with the design parameters for dynamic operation is the same as that of static operation; however, for light damping the magnitude of the peak EMCF will typically be an order of magnitude greater than that of static operation. For the actuator configuration considered in this study, it is shown that the absolute variation of EMCF with thickness ratio for dynamic operation is same as that for static operation when the damping ratio is 0.44 .
\end{abstract}

Keywords: Unimorph; Piezoelectric Actuators; Electromechanical coupling; Dynamics; Resonance. 


\section{Introduction}

Piezoelectric bending actuators are a class of actuators designed to utilise the inverse piezoelectric effect to convert input electric energy to output mechanical energy, and are capable of producing relatively large bending deflections at low voltage [1]. These actuators are constructed in different configurations and well known examples include unimorphs (actuators with a passive elastic layer and an active piezo-layer) and bimorphs (actuators with two active piezo-layers). Unimorphs are often preferred for their simple structure, ease of manufacture and improved reliability compared to bimorph structures [2,3]. Some common applications include laser mirror alignment, ink ejection in printing, and atomic force microscopy. Operation is typically performed off-resonance in order to prioritise positional accuracy over amplitude.

An alternative class of applications is one which achieves large displacements by operation at resonance. Examples of these resonant devices are piezoelectric actuated flapping-wing vehicles [2,4-8], fans [9-11] and pumps [12]. The current study is concerned with these engineering applications where the objective is to maximise fluid flow rate with respect to electrical power consumption. In order to properly address this challenge a detailed understanding of the underlying electromechanical properties of the actuator is necessary.

Existing theoretical treatments of piezoelectric actuators characterisation can be broadly divided into two cases: static and dynamic actuation. The evaluation of piezoelectric actuators performance in static operation is a well addressed problem in the literature. For static operation, the complete set of constituent equations relating the driving parameters (force and voltage) to the response parameters (deflection and charge) have been derived for unimorph and bimorph cantilever actuators [13] [14]. Wang et al. [3] used these equations to investigate actuator performance metrics as the electromechanical coupling factor and the maximum energy transmission coefficient. For unimorphs, they found that these metrics are related to the transverse coupling coefficient, $k_{31}$, the Young's modulus of the materials and the thickness ratio of the actuator layers, whereas for bimorphs it was found that dependency was on transverse coupling coefficient. 
In contrast to static actuation, the literature on dynamic performance of piezoelectric unimorph actuators still has significant gaps that require addressing. In the following paragraphs, some of the most relevant shortcomings within this aspect are highlighted. Smits and Ballato [15] provided the equations describing the dynamic behaviour of bimorph actuators in the form of a complete dynamic admittance matrix relating the harmonically varying driving parameters (tip moment, tip force, uniformly applied body force and voltage) to response parameters (tip rotation, tip deflection, volumetric displacement and charge). However, damping was ignored in their derivation and the work was not extended to the unimorph case. Later, dynamic structural models of unimorph actuators have been presented (e.g. [16]); however, only actuator mode shapes and natural frequencies were predicted. More recently, analytical models have been developed by Inman and his group taking damping into account [17-20]; however, these models concentrated on relating unimorph beam deflections to the applied voltage, and the models were not extended to include a dynamic admittance matrix relating the driving parameters to response parameters.

Actuator displacement, force and resonant frequency are important extrinsic metrics for actuation characterisation; however, the intrinsic metric provided by electromechanical coupling factor provides a more fundamental insight into the actuator performance for different applications (see discussion on this issue by Wang et al. [3], Rodriguez et al. [21] and Ikeda [22]). Thus, in the present work we focus the modelling and discussion on assessment of the electromechanical coupling factor rather than the higher level outputs of force and displacement. There have been some attempts to characterise the electromechanical coupling characteristics of unimorphs in dynamic operations. Basak et al. [16] developed analytical and finite element models to compute the open and short circuits natural frequencies of unimorphs from which they calculated the dynamic electromechanical coupling factor using the simple 'Mason formula' (a discussion on methods to evaluate the electromechanical coupling will be presented in section 2). A similar approach was also followed by Bidakhvidi et al. [23] to evaluate the dynamic electromechanical coupling factor of piezoelecric flapping wing propulsion structures. Chung et al. [6] demonstrated that the product of resonant frequency and vibration amplitude serves as a viable measure 
of unimorph performance. The same measure was applied later by Lal Kummari et al. [24] to the optimization of motion amplification mechanisms for flapping wing actuators. The use of the product of resonant frequency and vibration amplitude was based on an observation that this measure varies with the unimorph elastic/active layer thickness ratio in a similar fashion to the static energy transmission coefficient [6]. Therefore, the discussed methods can be regarded as indicatory rather than predictive, and there remains a need to develop a more comprehensive treatment of actuator dynamic characterisation that can be used for engineering design.

Despite these previous efforts, there exists no direct study to investigate and evaluate actuators electromechanical coupling factor in dynamic operation. As will be discussed in section 2, this requires the set of equations relating the driving parameters (force and voltage) to the response parameters (deflection and charge) to be derived. Thus in this study, a comprehensive extended analytical model is developed for the dynamic operation of unimorph actuators taking into account realistic operation conditions quantified by the damping ratio. The model is cast in the form of a matrix relating the harmonic driving parameters to response parameters allowing for the extraction of an analytical expression for the electromechanical coupling factor in dynamic operation. This allows for the first time analysis of the significant effect that damping can have on a piezoelectric actuator dynamic electromechanical coupling performance. The present work therefore provides insight into optimisation of the dynamic performance of unimorph actuators under realistic operating conditions through use of a comprehensive electromechanical dynamic model. This insight will ultimately enable the design of dynamic actuators with improved performance in terms of force and displacement output for a given input power. The next section will discuss the different measures of actuator performance represented through the definition of the electromechanical coupling factor. This will be followed by the development of a dynamic electromechanical model for unimorphs and its experimental verification. Finally, a comprehensive analysis of unimorph electromechanical coupling performance based on the theoretical model will be presented. 


\section{Electromechanical coupling factor}

The electromechanical coupling coefficient (EMCC, denoted by $k$ ) is a performance metric for a transducer; however, it is usually more meaningful to use the square of the EMCC, referred to as the electromechanical coupling factor (EMCF, $k^{2}$ ), which corresponds to the ratio of the stored mechanical energy to the supplied electrical energy $[22,3,21]$. Here we use the words coefficient and factor to distinguish between $k$ and $k^{2}$ respectively. Whilst EMCF is not a direct measure of the transducer efficiency, it represents a measure of the unavailable or ineffective fraction of energy [22] within an actuation cycle. Therefore, it serves as a useful measure to gauge piezoelectric actuator performance.

There are a number of different ways of deriving an expression for the EMCF. For a system close to resonance, a simple expression based on Mason's formula is given as [25,26,21]:

$$
k^{2}=\frac{f_{a}^{2}-f_{r}^{2}}{f_{a}^{2}},
$$

where $f_{r}$ is the resonant frequency and $f_{a}$ is the nearest anti-resonant frequency to $f_{r}$ determined from experimental measurements [26,21]. However, an alternative less restrictive expression for the EMCF can be obtained directly from the piezoelectric actuator equations according to Ikeda [22]. When the physical and electrical configuration of the piezoelectric actuator is defined, its behaviour is given by a set of constitutive equations derived in the form [3,22]:

$$
\left[\begin{array}{l}
\delta \\
Q
\end{array}\right]=\left[\begin{array}{ll}
C_{11} & C_{12} \\
C_{12} & C_{22}
\end{array}\right]\left[\begin{array}{l}
F \\
V
\end{array}\right],
$$

where $\delta, F, Q$ and $V$ are the tip deflection, tip force, charge and voltage, respectively. The EMCF is then defined as [3,22]:

$$
k^{2}=\frac{C_{12}^{2}}{C_{11} C_{22}},
$$


In the present study the dynamic EMCF of unimorph piezoelectric actuators will be derived from an analytical expression of Eqn. 2. In dynamic operation Eqn. 2 can be written as:

$$
\left[\begin{array}{l}
\delta(x, t) \\
Q(x, t)
\end{array}\right]=\left[\begin{array}{ll}
D_{1}(x) & D_{2}(x) \\
D_{3}(x) & D_{4}(x)
\end{array}\right]\left[\begin{array}{l}
F e^{i(\omega t)} \\
V e^{i(\omega t)}
\end{array}\right]
$$

where the real and imaginary parts of $e^{i \omega t}$ represent a cosine and sine form of excitation, respectively. Once derived, the elements of the dynamic matrix, $D_{i}(x)$, can be used in the same sense of Eqn. 3 to evaluate the dynamic EMCF of unimorph actuators. Whilst Eqn. 4 is presented in the form required for extraction of the EMCF, it also can be used to provide metrics for force and displacement using the first row that relates displacement, force and voltage, if required. 


\section{Electromechanical model}

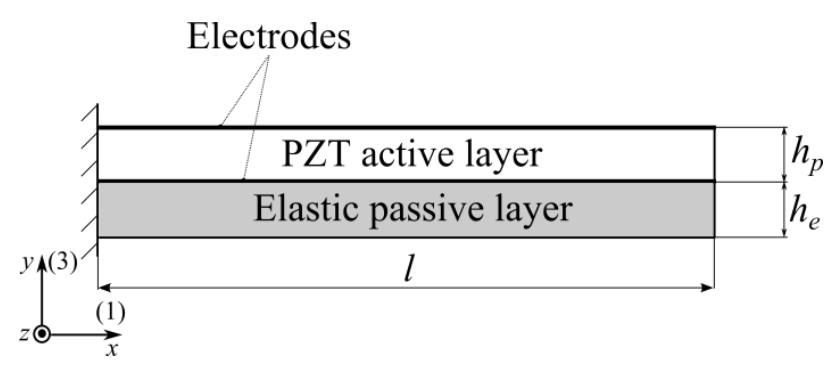

Figure (1): Schematic drawing of a piezoelectric unimorph bending actuator.

The derivation presented here builds on previous analytical treatments of bending actuators [17-20,27-29] especially the studies dealing with modelling the dynamic operation of unimorphs [17-20]. As discussed in the introduction, these studies [17-20] concentrated on modelling the relation between output unimorph deflection and input electric voltage. Nevertheless, they provided foundations that the present study utilises in deriving unimorph dynamic behaviour. We assume a unimorph bending actuator based on a uniform composite Euler-Bernoulli beam consisting of a PZT active layer perfectly bonded to an elastic passive layer, Fig. 1. The electrodes are assumed to be: (1) very thin (negligible thickness) compared to actuator layers; (2) perfectly conductive and (3) cover the entire PZT surface from top and bottom, so the electric field is uniform over the length [20]. The governing equation of motion can be written as $[29,20]$ :

$$
Y I \frac{\partial^{4} \delta(x, t)}{\partial x^{4}}+c \frac{\partial \delta(x, t)}{\partial t}+\rho A \frac{\partial^{2} \delta(x, t)}{\partial t^{2}}=f(x, t),
$$

where $Y$ is Young's modulus, $I$ is the area moment of inertia of the composite cross section, $c$ is the equivalent viscous damping coefficient that include damping sources, $\rho A$ is the mass per unit length, $f$ is the external applied force per unit length, $x$ is the spatial coordinate along the length of the actuator, $t$ is time and $\delta$ is the transverse deflection.

It should be noted that Eqn. 5 does not consider the Kelvin-Voigt damping term which requires a temporal derivative of the spatial fourth derivative. Viscous damping is influential at lower vibration modes, while Kelvin-Voigt damping is influential at higher vibration modes [20,30,31]. The main 
concern of the current model is towards applications such as flapping wing propulsion and fans. These applications operate at the first resonant frequency to achieve large displacements necessary for their functionality; therefore, it is appropriate to neglect the Kelvin-Voigt damping.

For a unimorph the rigidity, $Y I$, of the composite cross section is given by:

$$
Y I=\frac{w}{3}\left[Y_{e}\left(3 h_{e} \bar{y}\left(\bar{y}-h_{e}\right)+h_{e}^{3}\right)+Y_{p}\left(3 h_{p}\left(\bar{y}-h_{e}\right)\left(\bar{y}-\left(h_{e}+h_{p}\right)\right)+h_{p}^{3}\right)\right]
$$

where $w$ is the unimorph width, $h$ is the thickness and the subscripts $e$ and $p$ denote the elastic and PZT layers respectively. The neutral axis position, $\bar{y}$, is given by [13]:

$$
\bar{y}=\frac{\left(\frac{Y_{e}}{Y_{p}}\right) h_{e}^{2}+2 h_{e} h_{p}+h_{p}^{2}}{2\left(\left(\frac{Y_{e}}{Y_{p}}\right) h_{e}+h_{p}\right)},
$$

and the mass per unit length, $\rho A$, is given by:

$$
\rho A=\frac{m}{l}=w\left(\rho_{e} h_{e}+\rho_{p} h_{p}\right)
$$

where $m$ is the unimorph mass, $l$ is the length and $\rho$ is the material density. Finally, the damping coefficient, $c$, is given by:

$$
c=\rho A\left(2 \zeta_{n} \omega_{n}\right)
$$

where $\zeta$ is the damping ratio and $\omega$ is the natural frequency and $n$ indicates the $n$th mode.

For forced oscillations the steady state response of the beam can be obtained using modal analysis making use of the orthogonality of the mode shapes:

$$
\int_{0}^{l} X_{n}(x) X_{m}(x) d x=I_{n}=\int_{0}^{l} X_{n}^{2}(x) d x \text { for } n=m, \text { and is zero for } n \neq m
$$


The integral, $I_{n}$, can be obtained numerically once the mode shape, $X_{n}(x)$, is defined for given boundary conditions. Using the orthogonality property, the steady-state solution of Eqn. 5 is given by [19,27]:

$$
\delta(x, t)=\sum_{n=1}^{\infty} X_{n}(x) T_{n}(t)
$$

where $T_{n}(t)$ are determined from [27]:

$$
\frac{d^{2} T_{n}(t)}{d t^{2}}+2 \zeta_{n} \omega_{n} \frac{d T_{n}(t)}{d t}+\omega_{n}^{2} T_{n}(t)=\frac{1}{\rho A I_{n}} \int_{0}^{l} f(x, t) X_{n}(x) d x
$$

For a uniform cantilever beam the mode shapes are given by [29]:

$$
X_{n}(x)=\left(\cosh \left(\lambda_{n} x\right)-\cos \left(\lambda_{n} x\right)\right)-\sigma_{n}\left(\sinh \left(\lambda_{n} x\right)-\sin \left(\lambda_{n} x\right)\right)
$$

whose derivative with respect to $x$ is given by:

$$
\frac{d X_{n}(x)}{d x}=\lambda_{n}\left(\sinh \left(\lambda_{n} x\right)+\sin \left(\lambda_{n} x\right)\right)-\sigma_{n} \lambda_{n}\left(\cosh \left(\lambda_{n} x\right)-\cos \left(\lambda_{n} x\right)\right)
$$

where

$$
\sigma_{n}=\frac{\sinh \left(\lambda_{n} l\right)-\sin \left(\lambda_{n} l\right)}{\cosh \left(\lambda_{n} l\right)+\cos \left(\lambda_{n} l\right)}
$$

The clamped-free condition leads to the characteristic equation [29]:

$$
\cos (\lambda l) \cosh (\lambda l)=-1
$$

The first two roots of Eqn. 16 are $\lambda_{1}, l=1.8751$ and $\lambda_{2}, l=4.6941$ and the undamped natural frequency of the $n$th mode is given as:

$$
\omega_{n}=\frac{\left(\lambda_{n} l\right)^{2}}{l^{2}} \sqrt{\frac{Y I}{\rho A}}
$$

which is approximately the resonant frequency for light damping.

In order to obtain an expression for $D_{1}(x)$ in Eqn. 4 the force per unit length appearing in the right hand side of Eqn. 5 is expressed to represent an equivalent concentrated harmonic force at the actuator tip $[31,28]:$ 


$$
f(x, t)=F e^{i \omega t} \delta_{D}(x-l),
$$

where $\delta_{D}(x)$ is the Dirac delta function. Thus, the right hand side of Eqn. 12 becomes:

$$
\frac{1}{\rho A I_{n}} \int_{0}^{l} F e^{i \omega t} X_{n}(x) \delta_{D}(x-l) d x
$$

Since $F e^{i \omega t}$ is independent of $x$ it is taken out of the integration and using the Dirac delta function property:

$$
\int_{x_{l}}^{x_{u}} f(x) \delta_{D}(x-a) d x=f(a) ; \text { for } x_{l} \leq a \leq x_{u},
$$

the right hand side of Eqn. 12 becomes:

$$
\frac{F e^{i \omega t} X_{n}(l)}{\rho A I_{n}}=\frac{F e^{i \omega t}}{\rho A} \phi_{1}\left(\lambda_{n} l\right)
$$

where

$$
\phi_{1}\left(\lambda_{n} l\right)=\frac{X_{n}(l)}{I_{n}}
$$

Eqn. 22 can be evaluated numerically using the expression of mode shapes given by Eqn. 13. Substituting Eqn. 21 back into Eqn. 12 leads to:

$$
\frac{d^{2} T_{n}(t)}{d t^{2}}+2 \zeta_{n} \omega_{n} \frac{d T_{n}(t)}{d t}+\omega_{n}^{2} T_{n}(t)=F_{e q 1} e^{i \omega t}, F_{e q 1}=\frac{\phi_{1}\left(\lambda_{n} l\right)}{\rho A} F
$$

The particular (steady state) solution of the above differential equation is well known in the vibration literature as [29]:

$$
T_{n}(t)=A_{o} e^{i(\omega t-\theta)}, A_{o}=\frac{F_{e q 1}}{\omega_{n}^{2} \sqrt{\left(1-r_{n}^{2}\right)^{2}+\left(2 \zeta_{n} r_{n}\right)^{2}}}, \theta=\tan ^{-1} \frac{2 \zeta_{n} r_{n}}{1-r_{n}^{2}}, r_{n}=\frac{\omega}{\omega_{n}}
$$

Hence, following Eqn. 11 the first element of the dynamic matrix, $D_{1}(x)$, is obtained as: 


$$
D_{1}(x)=\sum_{n=1}^{\infty} X_{n}(x) \frac{\phi_{1}\left(\lambda_{n} l\right)}{\rho A} \frac{1}{\omega_{n}^{2} \sqrt{\left(1-r_{n}^{2}\right)^{2}+\left(2 \zeta_{n} r_{n}\right)^{2}}} .
$$

Now, the second element of the dynamic matrix, $D_{2}(x)$, will be derived. Bilgen et al. [18] showed that the right hand side of Eqn. 5 (i.e force per unit length) due to a voltage excitation of a unimorph bending actuator is given by:

$$
f(x, t)=\alpha V e^{i \omega t}\left[\frac{d \delta_{D}(x-l)}{d x}-\frac{d \delta_{D}(x)}{d x}\right]
$$

where

$$
\alpha=\frac{d_{31}}{h_{p}} \frac{Y_{p} w}{2}\left(h_{p}^{2}+2 h_{e} h_{p}-2 \bar{y} h_{p}\right)
$$

where $d_{31}$ is the piezoelectric constant. Following a similar procedure to that used to derive $D_{1}(x)$, Eqn. 26 is substituted into the right hand side of Eqn. 12 and making use of the Dirac delta function property:

$$
\int_{x_{l}}^{x_{u}} \frac{d^{n} \delta_{D}(x-a)}{d x^{n}} f(x) d x=(-1)^{n} \frac{d^{n} f(a)}{d x^{n}} ; \text { for } x_{l} \leq a \leq x_{u},
$$

the right hand side of Eqn. 12 becomes:

$$
-\frac{\alpha V e^{i \omega t}}{\rho A I_{n}} \frac{d X_{n}(l)}{d x}=-\frac{\alpha V e^{i \omega t}}{\rho A} \phi_{2}\left(\lambda_{n} l\right)
$$

where

$$
\phi_{2}\left(\lambda_{n} l\right)=\frac{1}{I_{n}} \frac{d X_{n}(l)}{d x}=\frac{\phi_{1}\left(\lambda_{n} l\right)}{X_{n}(l)} \frac{d X_{n}(l)}{d x}
$$

Equation 30 can be evaluated numerically using the expression of mode shapes given by Eqns 13 and 14 . Substituting Eqn. 29 back in Eqn. 12 leads to:

$$
\frac{d^{2} T_{n}(t)}{d t^{2}}+2 \zeta_{n} \omega_{n} \frac{d T_{n}(t)}{d t}+\omega_{n}^{2} T_{n}(t)=F_{e q 2} e^{i \omega t}, F_{e q 2}=-\frac{\alpha \phi_{2}\left(\lambda_{n} l\right)}{\rho A} V .
$$


Again the solution of the above equation is similar to that given in Eqn. 24; thus, the second element of the dynamic matrix, $D_{2}(x)$, is obtained:

$$
D_{2}(x)=-\sum_{n=1}^{\infty} X_{n}(x) \frac{\alpha \phi_{2}\left(\lambda_{n} l\right)}{\rho A} \frac{1}{\omega_{n}^{2} \sqrt{\left(1-r_{n}^{2}\right)^{2}+\left(2 \zeta_{n} r_{n}\right)^{2}}} .
$$

In order to obtain the expression of the third element of the dynamic matrix, $D_{3}(x)$, the relationship between charge, $Q$, and electric transverse displacement, $D_{3}$, is used [15,20,28]:

$$
Q=\int_{0}^{w} \int_{0}^{x} D_{3} d x d z
$$

An expression of the electric displacement can be obtained from the equations describing piezoelectricity $[15,20]:$

$$
\begin{aligned}
& S_{1}=\frac{1}{Y_{p}} T_{1}+d_{31} E_{3}, \\
& D_{3}=d_{31} T_{1}+\varepsilon_{33}^{T} E_{3},
\end{aligned}
$$

where $T$ is the stress, $S$ is the strain and $E$ is the electric field; subscripts 1 and 3 denote the $x$ and $y$ directions, respectively. Using the above equations it can be shown that the electric transverse displacement, $D_{3}$, is related to the piezoelectric strain, $S_{1}$, and the transverse electric field, $E_{3}$, by:

$$
D_{3}=Y_{p} d_{31} S_{1}+\left(\varepsilon_{33}^{T}-Y_{p} d_{31}^{2}\right) E_{3},
$$

with

$$
\varepsilon_{33}^{T}=\frac{d_{31}^{2} Y_{p}}{k_{31}^{2}}
$$

where $k_{31}$ is the piezoelectric material transverse electromechanical coupling coefficient [3] (a material property). Using the expression for $S_{l}$ of a unimorph actuator [20]: 


$$
S_{1}=\frac{-1}{2 h_{p}}\left(h_{p}^{2}+2 h_{e} h_{p}-2 \bar{y} h_{p}\right) \frac{\partial^{2} \delta}{\partial x^{2}}
$$

and substituting back with Eqns 35 and 37 into 33 leads to:

$$
Q=\int_{0}^{w} \int_{0}^{x}\left[Y_{p} d_{31}\left(\frac{-1}{2 h_{p}}\left(h_{p}^{2}+2 h_{e} h_{p}-2 \bar{y} h_{p}\right) \frac{\partial^{2} \delta}{\partial x^{2}}\right)+\left(\varepsilon_{33}^{T}-Y_{p} d_{31}^{2}\right) E_{3}\right] d x d z
$$

Integrating Eqn. 38 with respect to $x$ leads to:

$$
Q=\int_{0}^{w}\left[Y_{p} d_{31}\left(\frac{-1}{2 h_{p}}\left(h_{p}^{2}+2 h_{e} h_{p}-2 \bar{y} h_{p}\right) \frac{\partial \delta}{\partial x}\right)+\left(\varepsilon_{33}^{T}-Y_{p} d_{31}^{2}\right) E_{3} x\right] d z
$$

Performing the remaining integration and substituting the electric field as voltage divided by piezoelectric layer thickness gives:

$$
Q=\left(\frac{-d_{31} Y_{p} w}{2 h_{p}}\left(h_{p}^{2}+2 h_{e} h_{p}-2 \bar{y} h_{p}\right) \frac{\partial \delta}{\partial x}\right)+\left(\varepsilon_{33}^{T}-Y_{p} d_{31}^{2}\right) \frac{V(t)}{h_{p}} x w .
$$

Therefore, the electric charge is:

$$
Q=-\alpha \frac{\partial \delta}{\partial x}+\left(\varepsilon_{33}^{T}-Y_{p} d_{31}^{2}\right) \frac{V(t)}{h_{p}} x w
$$

In order to obtain the relation between tip force and charge, the displacement, $\delta$, caused by a tip force defined through the element $D_{1}(x)$ is substituted for in the above equation while setting the voltage to zero leading to:

$$
Q(x, t)=-\sum_{n=1}^{\infty} \frac{d X_{n}(x)}{d x} \frac{\alpha \phi_{1}\left(\lambda_{n} l\right)}{\rho A} \frac{1}{\omega_{n}^{2} \sqrt{\left(1-r_{n}^{2}\right)^{2}+\left(2 \zeta_{n} r_{n}\right)^{2}}} F e^{i(\omega t-\theta)} .
$$

Therefore, the third element of the dynamic matrix $D_{3}(x)$ is obtained:

$$
D_{3}(x)=-\sum_{n=1}^{\infty} \frac{d X_{n}(x)}{d x} \frac{\alpha \phi_{1}\left(\lambda_{n} l\right)}{\rho A} \frac{1}{\omega_{n}^{2} \sqrt{\left(1-r_{n}^{2}\right)^{2}+\left(2 \zeta_{n} r_{n}\right)^{2}}}
$$


Note that $D_{3}(l)$ has exactly the same value as $D_{2}(l)$, this can be shown from comparing Eqns 32 and 43 making use of Eqn. 30; therefore, the symmetry of the dynamic matrix is guaranteed at tip:

$$
D_{2}(l)=D_{3}(l)=D_{12}(l)
$$

Finally, to obtain the relationship between charge and voltage, the displacement, $\delta$, caused by voltage defined through the element $D_{2}(x)$ is substituted into Eqn. 41, leading to:

$$
Q(x, t)=\left[\sum_{n=1}^{\infty} \frac{d X_{n}(x)}{d x} \frac{\alpha^{2} \phi_{2}\left(\lambda_{n} l\right)}{\rho A} \frac{1}{\omega_{n}^{2} \sqrt{\left(1-r_{n}^{2}\right)^{2}+\left(2 \zeta_{n} r_{n}\right)^{2}}}+\left(\varepsilon_{33}^{T}-Y_{p} d_{31}^{2}\right) \frac{x w}{h_{p}}\right] V e^{i(\omega t-\theta)}
$$

Therefore, the fourth element of the dynamic matrix, $D_{4}(x)$, is obtained as:

$$
D_{4}(x)=\sum_{n=1}^{\infty} \frac{d X_{n}(x)}{d x} \frac{\alpha^{2} \phi_{2}\left(\lambda_{n} l\right)}{\rho A} \frac{1}{\omega_{n}^{2} \sqrt{\left(1-r_{n}^{2}\right)^{2}+\left(2 \zeta_{n} r_{n}\right)^{2}}}+\left(\varepsilon_{33}^{T}-Y_{p} d_{31}^{2}\right) \frac{x w}{h_{p}} .
$$

Whilst the above derivation concentrates on the modelling of unimorph cantilevered actuators, it can be extended to other boundary conditions (by changing mode shape representations) and/or actuator configurations (by changing configuration dependent parameters as $Y I, \bar{y}, \rho A, \alpha$ ). Thus, the work should be useful for researchers working with other piezoelectric configurations and under different boundary conditions. 


\section{Experimental verification}

In this section, an experimental setup to measure the dynamic actuation of unimorphs will be presented.

The objectives of this experiment are to: 1) identify the level of damping ratio required within the dynamic model; 2) validate possible aspects of the dynamic model given the available measurement facilities; 3) evaluate the EMCF experimentally making use of Mason's formula (Eqn. 1) and compare with the EMCF values obtained later in section 5 from the dynamic model.

The test specimens used in the current study are five unimoph actuators of the same width and thickness, but different lengths, Fig. 2a. The elastic layer is brass and the active layer is PZT (Murata PIEZOTITE ceramic, P-7 [32]). The test specimens were manufactured from commercially available diaphragms (Murata Manufacturing Co., Ltd., Part number: 7BB-35-3L0 [32]). This provides a fast and cost effective way to prepare beams of different lengths; however, it limits the ability to vary the thicknesses of the elastic and PZT layers. Table 1 provides typical properties of the prepared unimorph beams.

Table (1): Typical properties of the unimorph beams.

\begin{tabular}{ll}
\hline Geometric properties & \\
\hline Length, $l(\mathrm{~mm})$ & $16,18,20,22,24$ \\
Width, $w(\mathrm{~mm})$ & 4.5 \\
Elastic layer thickness, $h_{e}(\mathrm{~mm})$ & 0.3 \\
Active layer thickness, $h_{p}(\mathrm{~mm})$ & 0.23 \\
\hline Elastic layer material properties & \\
\hline Material & Brass \\
Young's modulus, $Y_{e}(\mathrm{GPa})$ & 110 \\
Density, $\rho_{e}\left(\mathrm{~kg} / \mathrm{m}^{3}\right)$ & 8600 \\
\hline Active layer material properties & \\
\hline Model & Murata PIEZOTITE ceramic \\
Type & PZT, P-7 \\
Young's modulus, $Y_{p}(\mathrm{GPa})$ & 63 \\
Density, $\rho_{p}\left(\mathrm{~kg} / \mathrm{m}^{3}\right)$ & 7800 \\
Electromechanical coupling, $k_{31}$ & 0.38 \\
Piezoelectric constant, $d_{31}\left(10^{-12} \mathrm{~m} / \mathrm{V}\right)$ & -207 \\
Maximum voltage $(\mathrm{V})$ & 15 \\
\hline
\end{tabular}


Figure $2 \mathrm{~b}$ shows a schematic of the experimental apparatus. Beam oscillation displacement was measured using a Polytec PDV-100 laser vibrometer. The input sinusoidal signal to the beams was generated using a TTi TG200 20 MHz DDS function generator amplified using a TREK PZD350 Piezo Driver/Amplifier. The measurement setup was managed through LabVIEW. Data acquisition was conducted within the frequency range appropriate for each measurement/beam combination with $1 \mathrm{~Hz}$ resolution.

(a)

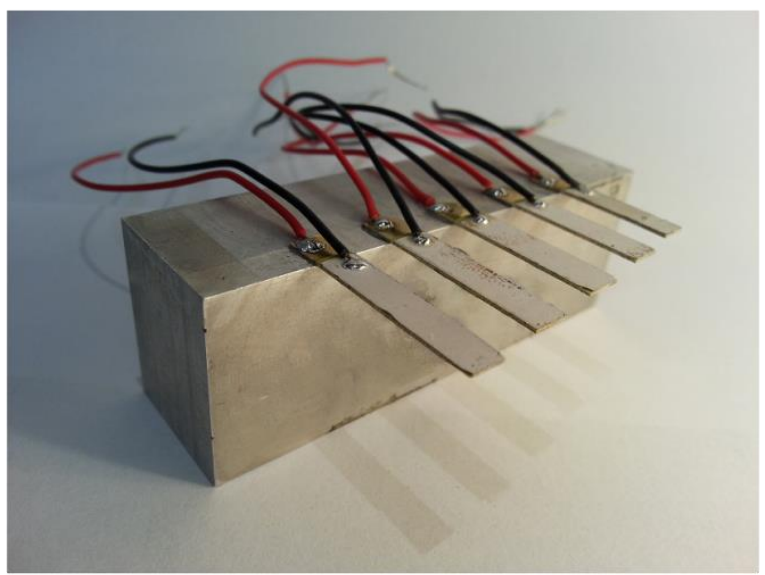

(b)

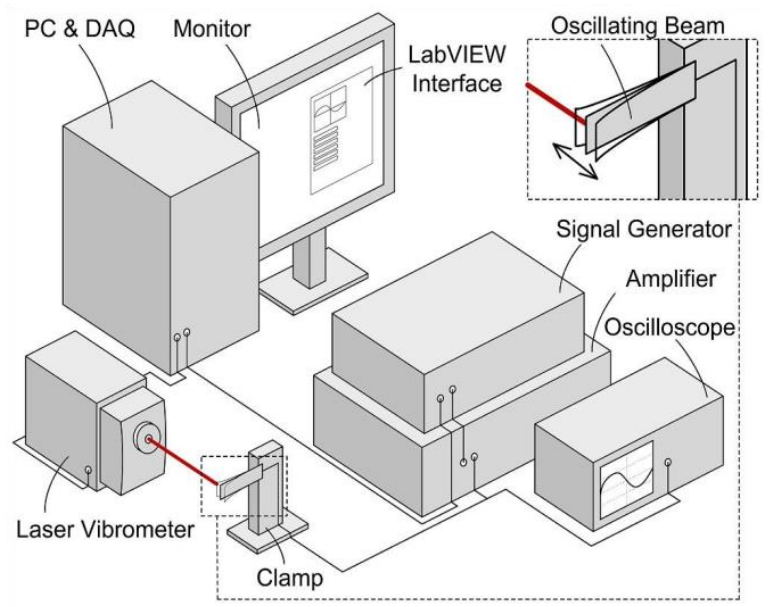

Figure (2): (a) Unimorph cantilever beams used during the measurements. Each beam is $4.5 \mathrm{~mm}$ wide. (b) schematic of the used measurement setup. 
Firstly, the fundamental frequency and associated mode shape were measured for each beam, Figure 3. A comparison of the measured first resonant frequency with that evaluated from the model (Eqn. 17) is shown in Fig. 3a. Also, Fig. 3b shows the experimental and theoretical mode shapes for the $24 \mathrm{~mm}$ beam. The theoretical mode shape is obtained from Eqn. 13, and the experimental mode shape is obtained by measuring the amplitude of the deflection along the beam while operating around the first resonant frequency. Both results show good agreement between the model and the experimental measurements.

(a)

(b)
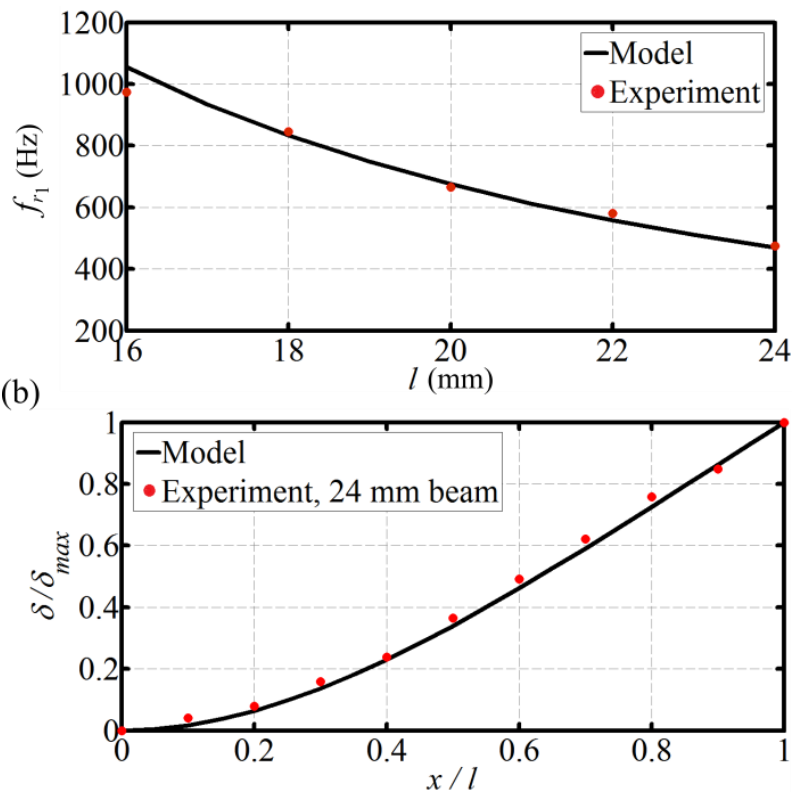

Figure (3): (a) Fundamental bending resonant frequency variation with the beam length having all other variables fixed. (b) Fundamental mode shape of the $24 \mathrm{~mm}$ beam; $\delta$ is the amplitude of deflection along the beam length and $\delta_{\max }$ is the amplitude of deflection at the beam tip.

Next, measurements were conducted to characterise the damping ratio based on an evaluation of the harmonic vibration response, Eqn. 47.

$$
\zeta_{1}=\frac{\left(f_{2}-f_{1}\right)}{2 f_{r 1}},
$$

where $f_{r 1}$ is the first resonant frequency, and $f_{1}$ and $f_{2}$ are the half-power point frequencies where the response is 0.707 of the maximum [27]. 
Figure 4a shows the measured tip velocity in the range around the first resonant frequency for the 16 $\mathrm{mm}$ beam at different input voltage amplitudes. The response is weakly nonlinear with voltage, with the first resonant frequency decreasing as the voltage increases. This observation was also reported by Wang et al. [33]. The damping ratios for the $16 \mathrm{~mm}$ beam were higher than those of other beams. For this reason the $16 \mathrm{~mm}$ beam results are used to exemplify the variation of damping ratio with voltage in Fig. $4 \mathrm{~b}$. For this case, the damping ratio increases with increasing voltage, and varies between 0.011 and 0.022 .
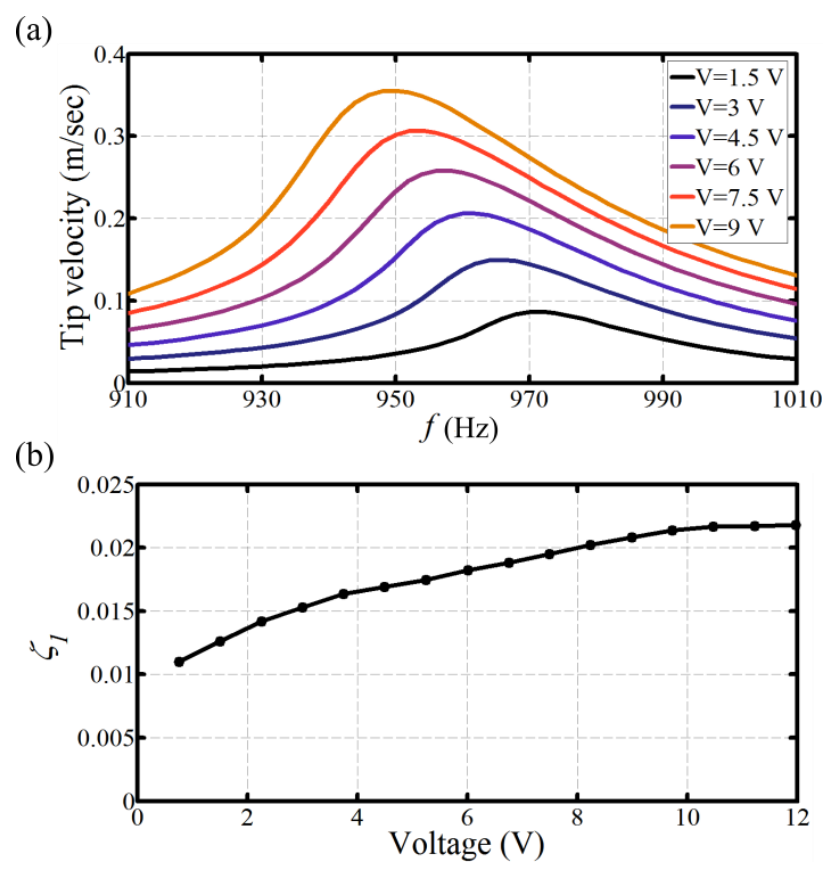

Figure (4): (a) Measured tip velocity of the $16 \mathrm{~mm}$ actuator plotted against driving frequency at several driving voltage amplitudes. (b) Variation of damping ratio with voltage amplitude for the $16 \mathrm{~mm}$ beam. Note that, an input signal greater than $12 \mathrm{~V}$ yields tip velocities higher than the measurable limits of the vibrometer.

Having obtained the damping coefficient values at the different voltage levels, the second element of the dynamic matrix, $D_{2}(x)$, can now be used to relate the driving voltage to the output displacement. Figure 5a compares the measured tip deflection amplitude of the $24 \mathrm{~mm}$ unimorph due to a 7.5 voltage amplitude signal with that evaluated from the theoretical model using the expression of $D_{2}(l)$. Over the range of frequencies considered, a very good agreement is evident. Figure $5 \mathrm{~b}$ compares the measured and modelled tip deflection amplitude at the fundamental bending resonant frequency as a function of the 
input voltage amplitude for the $16 \mathrm{~mm}$ and $24 \mathrm{~mm}$ unimorphs. Note that the resonant frequency of the beam shifts slightly with the voltage amplitude (Figure $4 a$ ); thus, the experimental values in Fig. $5 \mathrm{~b}$ are the measured beam tip deflection values at the fundamental resonant frequency corresponding to each voltage amplitude.
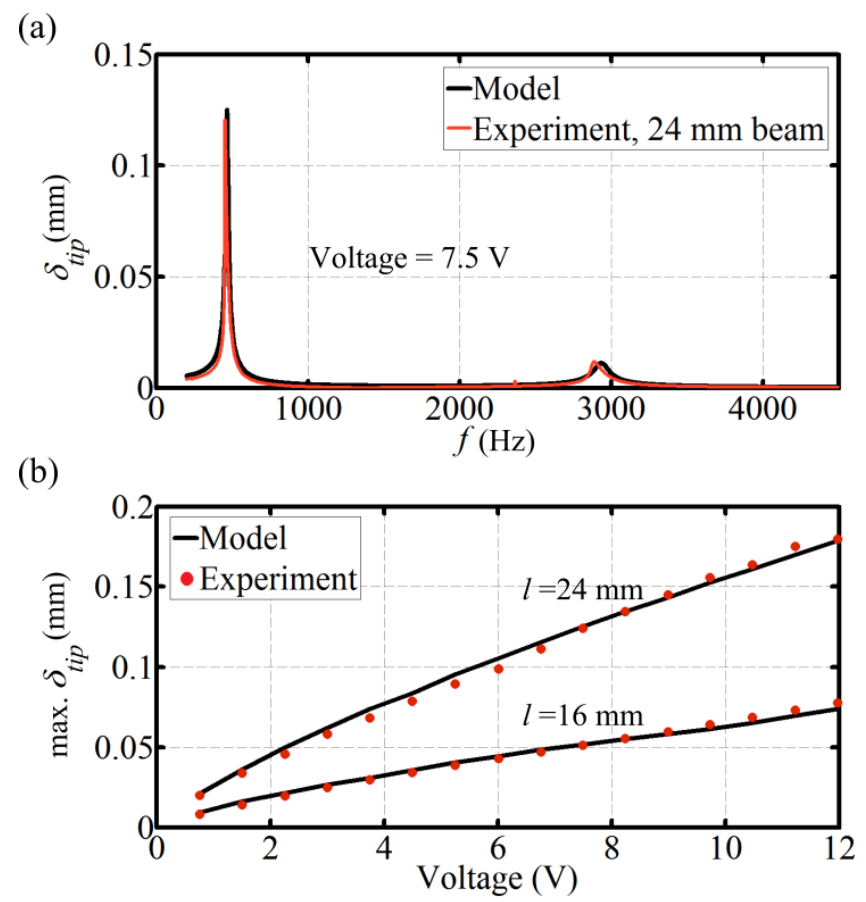

Figure (5): (a) Tip deflection amplitude of the $24 \mathrm{~mm}$ actuator for the first two modes. (b) Tip deflection amplitude of the $16 \mathrm{~mm}$ and $24 \mathrm{~mm}$ actuators at their fundamental (first) resonant frequency as a function of the input voltage amplitude.

Finally, the tip velocity to harmonic voltage excitation frequency response function (FRF) was measured for each of the five beam specimens, with the objective of determining the anti-resonant frequencies required in Mason's formula (Eqn. 1). Figure 6 shows the FRF for measurements with an input voltage amplitude of $7.5 \mathrm{~V}$, representing a mid-range value for the input signal. 


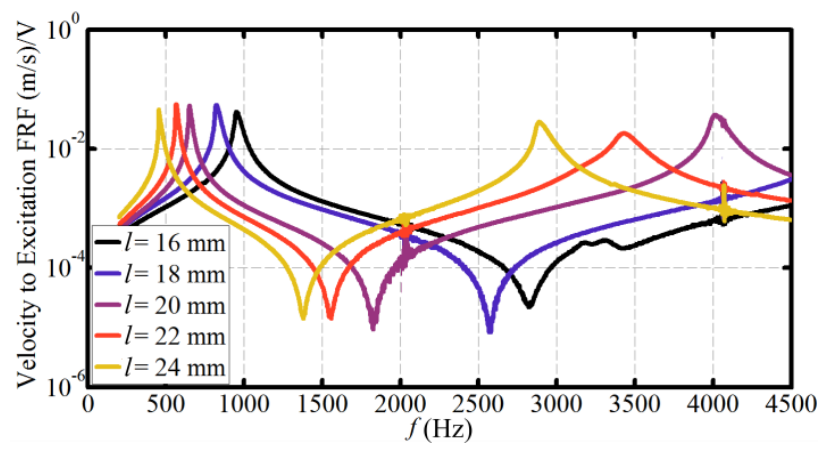

Figure (6): Experimental tip velocity to harmonic voltage excitation FRF of the five beams. The voltage level is $7.5 \mathrm{~V}$ for all beams.

Table 2 shows that the variation of the EMCF using Eqn. 1 for the different beam specimens is minimal and an average value of $0.88 \pm 0.013$ (mean \pm s.d.) can be assigned for all beams. Note that varying the voltage level changes the resonant and anti-resonant frequencies slightly; however, the EMCF value from Mason's formula remains almost unchanged. The important outcome here is that the average value for the dynamic EMCF is significantly higher than that for static operation. This will be discussed in the next section, which will focus on evaluating the dynamic EMCF for unimorphs using the derived dynamic model.

Table (2): Unimorph actuators EMCF at excitation level of $7.5 \mathrm{~V}$.

\begin{tabular}{cccc}
\hline $\begin{array}{c}l \\
(\mathrm{~mm})\end{array}$ & $\begin{array}{c}f_{r 1} \\
(\mathrm{~Hz})\end{array}$ & $\begin{array}{c}f_{a 1} \\
(\mathrm{~Hz})\end{array}$ & $\begin{array}{c}k^{2} \\
\text { Eqn. } 1\end{array}$ \\
\hline 16 & 954 & 2822 & 0.88 \\
18 & 824 & 2568 & 0.9 \\
20 & 650 & 1826 & 0.87 \\
22 & 566 & 1558 & 0.87 \\
24 & 454 & 1380 & 0.89 \\
\hline
\end{tabular}




\section{Analysis of the electromechanical coupling in dynamic operation}

The electromechanical coupling factor, $k^{2}$, was discussed comprehensively in Section 2 as important indicator for piezoelectric device performance. Using the complete dynamic matrix for unimorph actuators from Section 3, Eqns 3 and 4 can now evaluate the EMCF in dynamic operation as:

$$
k^{2}=\frac{D_{12}^{2}(l)}{D_{1}(l) D_{4}(l)} .
$$

It should be noted that the results presented in this section are all based on the theoretical model of section 3. Within the theoretical model, the material properties and damping levels will be selected to be similar to those from the experiments. The dynamic $k^{2}$ from Eqn. 48 will be evaluated at the first resonant frequency as this condition provides maximum tip deflection which is consistent with the objective of many practical applications.

Following Eqn. 48, the current analysis shows that the dynamic EMCF of unimorphs depends only on: 1) the Young's modulus of the elastic and active layers, 2) the thicknesses of the elastic and active layers, 3) the damping ratio, $\zeta$ and 4) the PZT layer transverse electromechanical coupling coefficient, $k_{31}$ (material property). In what follows, the influence of each of these four factors on the dynamic EMCF will be discussed in detail.

Table (3): Unimorph passive layer material properties.

\begin{tabular}{ccc}
\hline Material & $\begin{array}{c}Y_{e} \\
(\mathrm{GPa})\end{array}$ & $\begin{array}{c}\rho_{e} \\
\left(\mathrm{~kg} / \mathrm{m}^{3}\right)\end{array}$ \\
\hline Steel & 195 & 7800 \\
Brass & 110 & 8600 \\
Aluminium & 65 & 2700 \\
\hline
\end{tabular}


(a)

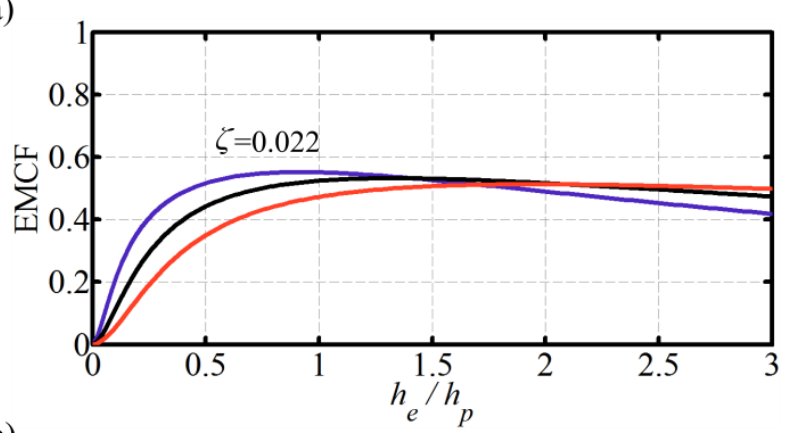

(b)

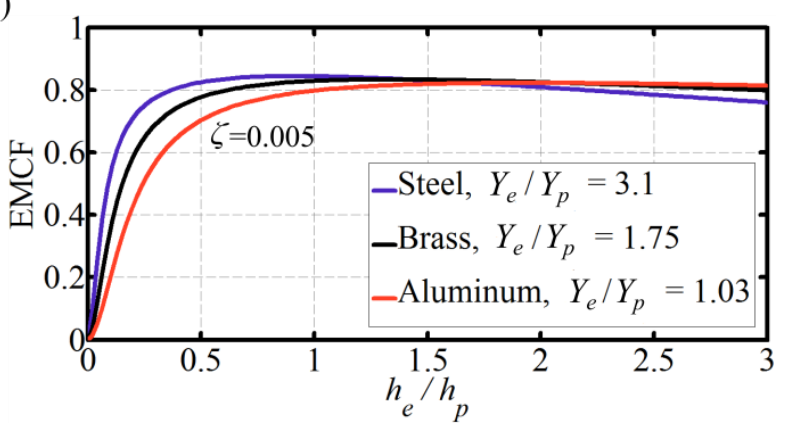

Figure (7): Dynamic EMCF as function of (passive/active) layer thickness ratio for different passive layer materials (a) damping ratio of 0.022 . (b) damping ratio of 0.005 . In both figures the active layer material properties are $Y_{p}=63 \mathrm{GPa}$ and $k_{31}=0.38$.

For the current analysis, unimorphs made of the same active layer but with different passive layer materials are considered (Table 3); the active layer material properties used are $Y_{p}=63 \mathrm{GPa}$ and $k_{31}=0.38$. Figure 7 shows the dynamic EMCF plotted against elastic/piezo thickness ratio for different elastic materials for a damping ratio of 0.022 (Figure 7a) and a damping ratio of 0.005 (Figure 7b). Note that a damping ratio of 0.022 represents the higher end of damping obtained from experiments. The overall trend is a rapid increase in EMCF as the thickness ratio increases from zero, with a peak reached at an optimum thickness ratio; beyond this, the EMCF decreases slightly as the thickness ratio increases. The effect of increasing elastic material stiffness is to shift the thickness ratio for peak EMCF (optimum thickness ratio) to the left. This optimum thickness ratio is found to vary only with the Young's modulus ratio of the elastic and active layers, Fig. 8. Decreasing damping ratio increases the peak value of EMCF however does not affect the thickness ratio at which it occurs. Stiffer elastic material leads to higher peak EMCF values; however, at very low damping ratios, the peak EMCF for different stiffness ratio values are relatively closer. 


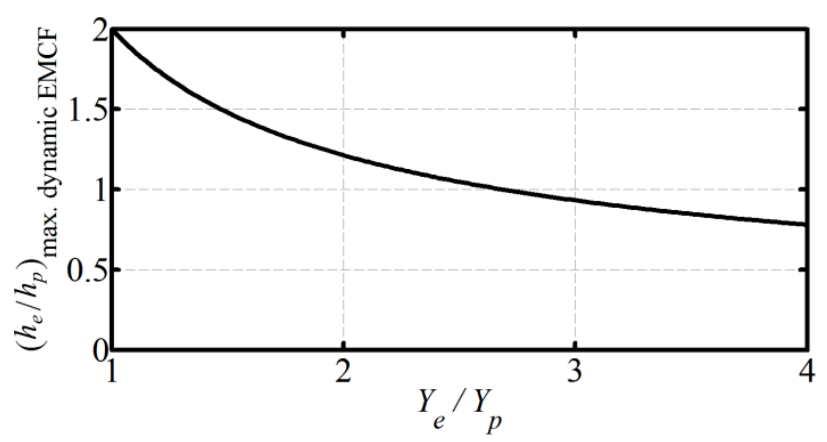

Figure (8): Dynamic operation optimum thickness ratio variation with the Young's modulus ratio.

To further define the effect of damping Fig. 9a shows the dynamic EMCF for a unimorph (elastic layer: brass; active layer with $Y_{p}=63 \mathrm{GPa}$ ) as a function of thickness ratio for three different damping ratios. A similar analysis using several materials but based on the static equations showed that a typical unimorph actuator can only achieve EMCF values up to 0.08 [3], which is an order of magnitude less than the peak EMCF values shown here. This highlights the important role damping plays in determining the achievable performance of such actuators in dynamic operation: unimorph actuators achieve high dynamic EMCF values when subjected to light damping. These high EMCF values are similar to EMCF values obtained experimentally using Mason's formula.

The effect of the PZT layer transverse electromechanical coupling coefficient on the dynamic EMCF is similar to the damping ratio effect, Fig. 9b. It also scales the EMCF curve up (higher $k_{31}$ ) and down (lower $k_{31}$ ) without influencing the EMCF shape variation against the (elastic/active) thickness ratio. This is demonstrated for a range of values of electromechanical coupling coefficients that is typical of currently available actuators. 

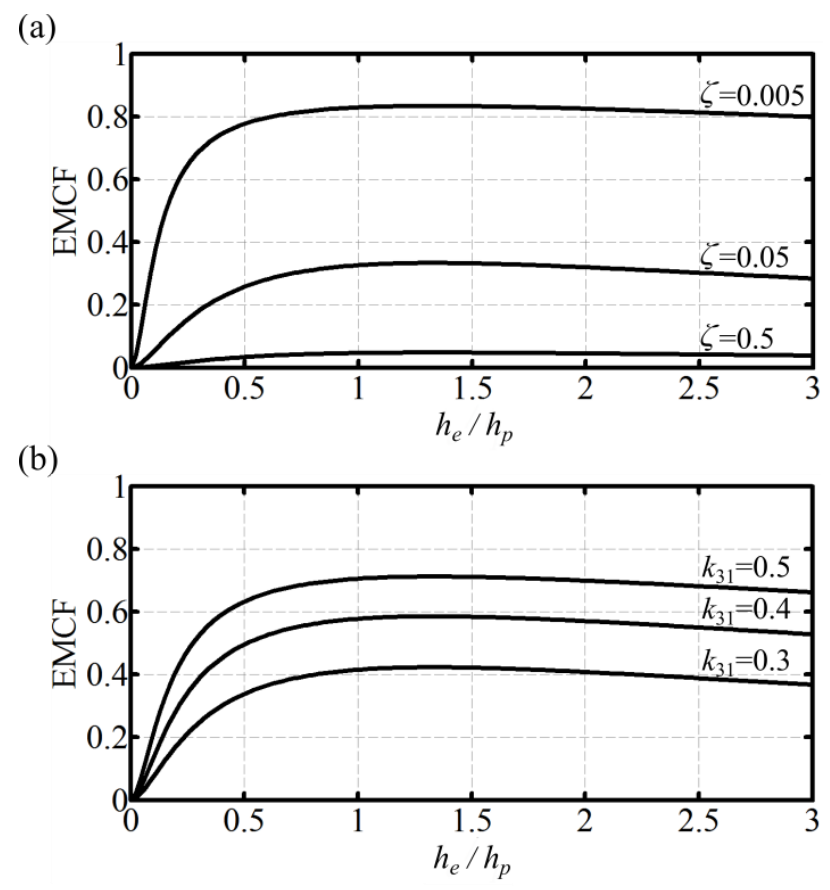

Figure (9): Dynamic EMCF as function of (passive/active) layer thickness ratio for a brass passive layer and an active layer of $Y_{p}=63 \mathrm{GPa}$. (a) Effect of damping ratio, $k_{31}=0.38$. (b) Effect of the PZT layer transverse electromechanical coupling coefficient, $\zeta=0.02$.

Lastly, the static and dynamic EMCF at equivalent conditions are compared. There is a damping ratio at which the unimorph dynamic electromechanical coupling is equal to the static one. Figure 10 compares the static EMCF (based on equations in Appendix A) with the dynamic EMCF at a damping ratio of 0.44 for a unimorph of an elastic layer from brass and an active layer with $Y_{p}=63 \mathrm{GPa}$. The two curves are the same and have the same peak value showing that for practical purposes the optimum thickness ratio for static operation is the same as that for dynamic operation.

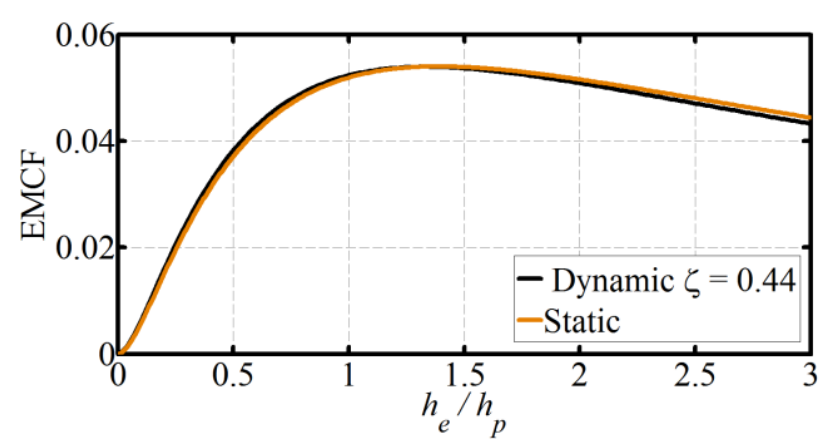

Figure (10): EMCF plotted for a unimoph at static and equivalent dynamic condition. The unimorph is made of an elastic brass layer and an active layer with $Y_{p}=63 \mathrm{GPa}$ and $k_{31}=0.38$. 


\section{Conclusions}

A comprehensive analytical model of the dynamic electromechanical behaviour of a piezoceramic unimorph actuator has been derived and successfully validated against experimental data. An analytical expression for the EMCF extracted from the model is used as the objective for parametric design studies. It is found that the shape of variation of EMCF with thickness ratio for a given unimorph geometry and material properties is the same for both static and dynamic operation, meaning that an actuator optimised for static operation with respect to maximising EMCF will also be optimal for dynamic operation. However, the achievable values of the EMCF for dynamic operation are dependent on damping ratio.

A peak value of EMCF occurs at an optimal thickness ratio that varies only with the Young's modulus ratio of the elastic and active layers for the actuator configuration considered in this study. Increasing elastic layer material Young's modulus shifts the optimal thickness ratio to the left (thinner elastic layer). Increasing the thickness ratio beyond the optimal value (increasing elastic layer thickness) has a relatively minor effect on the achievable EMCF for practical actuator configurations. The achievable magnitude of EMCF is increased by decreasing the damping ratio and/or increasing $k_{31}$. However, the thickness ratio for given material properties at which maximum EMCF is achieved is independent of both damping ratio and $k_{31}$. As the actuator damping is reduced towards zero, EMCF is decreasingly sensitive to thickness ratio for practical actuator configurations and tends towards a value of unity. 


\section{Appendix (A):}

These equations were used for the evaluation of the static curve in Fig. 10. The static actuation constituent equations governing unimorphs are given as $[3,13]$ :

$$
\left[\begin{array}{l}
\delta \\
Q
\end{array}\right]=\left[\begin{array}{ll}
S_{1} & S_{2} \\
S_{3} & S_{4}
\end{array}\right]\left[\begin{array}{l}
F \\
V
\end{array}\right] .
$$

where

$$
\begin{gathered}
S_{1}=\frac{4 s_{11}^{p} l^{3}}{w h_{p}^{3}} \frac{A B+1}{1+4 A B+6 A B^{2}+4 A B^{3}+A^{2} B^{4}}, \\
S_{2}=S_{3}=\frac{3 d_{31} l^{2}}{h_{p}^{2}} \frac{A B(B+1)}{1+4 A B+6 A B^{2}+4 A B^{3}+A^{2} B^{4}}, \\
S_{4}=\frac{\varepsilon_{33}^{T} l w}{h_{p}}\left(1-k_{31}^{2} \frac{A B\left(1+A B^{3}\right)}{1+4 A B+6 A B^{2}+4 A B^{3}+A^{2} B^{4}}\right) \\
A=\frac{s_{11}^{p}}{s_{11}^{e}}=\frac{Y_{e}}{Y_{p}}, B=\frac{h_{e}}{h_{p}} .
\end{gathered}
$$

\section{List of notations:}

$c$

$d_{31}$

$D_{3}$

$D_{i}(x)$

$E_{3}$

$f(x, t)$

$f_{1}, f_{2}$

$f_{a}$

$f_{r}$

F

h

I

$k$

$k^{2}$

$k_{31}$
: viscous damping coefficient

: piezoelectric constant

: electric displacement in 3-direction

: $i^{\text {th }}$ element of the dynamic matrix

: electric field in 3-direction

: external force per unit length

: half-power point frequencies

: anti-resonant frequency

: resonant frequency

: force

: thickness

: area moment of inertia of the composite cross section

: electromechanical coupling coefficient, EMCC

: electromechanical coupling factor, $\mathrm{EMCF}$

: piezoelectric material electromechanical coupling coefficient 


\begin{tabular}{|c|c|c|}
\hline$l$ & $:$ & length \\
\hline$m$ & : & mass \\
\hline$Q$ & : & electric charge \\
\hline$s_{11}$ & : & elastic compliance \\
\hline$S_{1}$ & : & piezoelectric strain in 1-dirction \\
\hline$S_{i}$ & : & $i^{\text {th }}$ element of the static matrix \\
\hline$t$ & : & time \\
\hline$T_{n}(t)$ & : & time solution function \\
\hline$T_{1}$ & : & piezoelectric stress in 1-dirction \\
\hline$V$ & : & voltage \\
\hline$w$ & : & width \\
\hline$x$ & : & spatial coordinate along actuator length \\
\hline$X_{n}(x)$ & $:$ & mode shape \\
\hline$y$ & $:$ & spatial coordinate along actuator thickness \\
\hline $\bar{y}$ & $:$ & neutral axis position \\
\hline$Y$ & : & Young's modulus \\
\hline$z$ & : & spatial coordinate along actuator width \\
\hline \multicolumn{3}{|c|}{ Greek Symbols } \\
\hline$\delta$ & : & transverse deflection \\
\hline$\delta_{D}$ & : & Dirac delta function \\
\hline$\varepsilon_{33}^{T}$ & : & permittivity at constant stress \\
\hline$\rho$ & : & density \\
\hline$\rho A$ & : & mass per unit length \\
\hline$\omega$ & : & frequency \\
\hline$\zeta$ & : & damping ratio \\
\hline \multicolumn{3}{|c|}{ Subscripts } \\
\hline 1 & : & denotes length direction \\
\hline 3 & : & denotes thickness direction \\
\hline$e$ & : & elastic \\
\hline$e q$ & : & equivalent \\
\hline$n$ & : & indicates $n^{\text {th }}$ mode shape \\
\hline$p$ & : & PZT \\
\hline \multicolumn{3}{|c|}{ Abbreviations } \\
\hline EMCC & : & Electromechanical Coupling Coefficient \\
\hline EMCF & : & Electromechanical Coupling Factor \\
\hline PZT & : & Lead Zirconate Titanate (stands for piezoelectric) \\
\hline
\end{tabular}

\section{Acknowledgement}

The authors would like to thank Andrew Kennaugh and Khristopher Kabbabe from the University of Manchester for helping with the experimental setup.

This research received no specific grant from any funding agency in the public, commercial, or not-forprofit sectors. 


\section{References:}

1. Wang Q, Du X, Xu B and Cross L. Theoretical analysis of the sensor effect of cantilever piezoelectric benders. J. Appl. Phys. 1999; 85: 1702-1712.

2. Sitti M, Campolo D, Yan J, Fearing RS, Su T, Taylor D and Sands TD. Development of PZT and PZN-PT based unimorph actuators for micromechanical flapping mechanisms. Proceedings of the IEEE International Conference on Robotics and Automation, Seoul, Korea, May 2001, 3839-3846.

3. Wang Q, Du X, Xu B and Cross L. Electromechanical coupling and output efficiency of piezoelectric bending actuators. IEEE Trans. Ultrason. Ferroelect. Freq. Contr. 1999; 46: 638-646.

4. Cox A, Monopoli D, Cveticanin D, Goldfarb M and Garcia E. The development of elastodynamic components for piezoelectrically actuated flapping micro air vehicles. J. Intell. Mater. Syst. 2002; 13: 611-615.

5. Syaifuddin M, Park HC and Goo NS. Design and evaluation of a LIPCA-actuated flapping device. Smart. Mater. Struct. 2006; 15: 1225- 1230.

6. Chung HC, Kummari KL, Croucher SJ, Lawson NJ, Guo S, Whatmore RW and Huang Z. Development of piezoelectric fans for flapping wing application. Sens. Actuators. A Phys. 2009; 149: 136-142.

7. Mukherjee S and Ganguli R. Non-linear dynamic analysis of a piezoelectrically actuated flapping wing. J. Intell. Mater. Syst. Struct. 2010; 21: 1157-1167.

8. $\mathrm{Hu} \mathrm{H}$, Clemons L and Igarashi $\mathrm{H}$. An experimental study of the unsteady vortex structures in the wake of a root-fixed flapping wing. Exp Fluids 2011; 51: 347-359.

9. Yoo J, Hong J and Cao W. Piezoelectric ceramic bimorph coupled to thin metal plate as cooling fan for electronic devices. Sens. Actuators. A Phys. 2000; 79: 8-12.

10. Yao K and Uchino K. Analysis on a composite cantilever beam coupling a piezoelectric bimorph to an elastic blade. Sens. Actuators. A Phys. 2001; 89: 215-221. 
11. Wu T, Ro P, Kingon A and Mulling J. Piezoelectric resonating structures for microelectronic cooling. Smart. Mater. Struct. 2003; 12: 181-187.

12. de Lima C, Vatanabe S, Choi A, Nakasone P, Pires R and Silva E. A biomimetic piezoelectric pump: Computational and experimental characterization. Sens. Actuators. A Phys. 2009; 152: 110-118.

13. Smits JG and Choi W-S. The constituent equations of piezoelectric heterogeneous bimorphs. IEEE Trans. Ultrason. Ferroelect. Freq. Contr. 1991; 38: 256-270.

14. Smits JG, Dalke SI and Cooney TK. The constituent equations of piezoelectric bimorphs. Sens. Actuators. A Phys. 1991; 28: 41-61.

15. Smits JG and Ballato A. Dynamic admittance matrix of piezoelectric cantilever bimorphs. J. Microelectromech. Syst. 1994; 3: 105-112.

16. Basak S, Raman A and Garimella SV. Dynamic response optimization of piezoelectrically excited thin resonant beams. J. Vib. Acoust. 2005; 127: 18-27.

17. Bilgen O, Erturk A and Inman DJ. Analytical and experimental characterization of macro-fiber composite actuated thin clamped-free unimorph benders. J. Vib. Acoust. 2010; 132: 051005.

18. Bilgen O, Karami MA, Inman DJ and Friswell MI. The actuation characterization of cantilevered unimorph beams with single crystal piezoelectric materials. Smart. Mater. Struct. 2011; 20: 1-9.

19. Bilgen O, Wang Y and Inman DJ. Electromechanical comparison of cantilevered beams with multifunctional piezoceramic devices. Mech. Syst. Signal Process. 2012; 27: 763-777.

20. Erturk A and Inman DJ. A distributed parameter electromechanical model for cantilevered piezoelectric energy harvesters. J. Vib. Acoust. 2008; 130: 041002.

21. Rodríguez H, Ceres R, Calderon L, and Pons JL. Modelling of the travelling wave piezoelectric motor stator: an integrated review and new perspective. Boletín de la Sociedad Española de Cerámica y Vidrio. 2004; 43: 698-705.

22. Ikeda T. Fundamentals of piezoelectricity. Oxford University Press, 1996. 
23. Bidakhvidi MA, Vucinic D and Vanlanduit S. Multiphysics optimization of the piezoelectric flapping wing propulsion. International conference on Engineering Optimization. Rio de Janeiro, Brazil, 1-5 July 2012.

24. Lal Kummari K, Li D, Guo S and Huang Z. Development of piezoelectric actuated mechanism for flapping wing micro-aerial vehicle applications. Adv. Appl. Ceram. 2010; 109: 175-179.

25. Mason WP. Piezoelecric crystals and their application to ultrasonics. New York Van Nostrand, 1950.

26. Chang SH, Rogacheva NN and Chou CC. Analysis of methods for determining electromechanical coupling coefficients of piezoelectric elements. IEEE Trans. Ultrason. Ferroelect. Freq. Contr. 1995; 42: 630-640.

27. Braun S, Ewins D and Rao SS. Encyclopedia of vibration. Vol. 1, Academic Press, 2002.

28. Ballas RG. Piezoelectric multilayer beam bending actuators: static and dynamic behavior and aspects of sensor integration. Springer, 2007.

29. Inman DJ. Engineering vibrations. 3rd ed. Pearson International Edition, 2009.

30. Renardy, M. On localized Kelvin-Voigt damping. ZAMM-Journal of Applied Mathematics and Mechanics. 2004; 84: 280-283.

31. Abu-Hilal, M. Forced vibration of Euler-Bernoulli beams by means of dynamic Green functions. J. Sound Vib. 2003; 267: 191-207.

32. Catalog No. P19E-6, Piezoelectric Ceramic Sensors (PIZOTITE ${ }^{\circledR}$ ), Murata Manufacturing Co., Ltd.

33. Wang Q, Zhang Q, Xu B, Liu R and Cross LE. Nonlinear piezoelectric behavior of ceramic bending mode actuators using strong electric fields. J. Appl. Phys. 1999; 86: 3352-3360. 